\title{
Survey of marketable vegetables and edible fruits in Dharan, eastern Nepal
}

\author{
Sabitri Shrestha ${ }^{1^{*}}$ and Shiva Kumar Rai ${ }^{2}$ \\ ${ }^{1}$ Department of Biology, Central Campus of Technology, Dharan, T.U., Nepal \\ ${ }^{2}$ Department of Botany, P.G. Campus, T.U., Biratnagar, Nepal \\ *E-mail: sabli123123@yahoo.co.in
}

\begin{abstract}
A total 77 types of vegetables and 33 fruits were recorded from the markets of Dharan during the period of one year. Among them, 11 vegetables viz. Agaricus bisporus, Allium cepa, A. sativum, Capsicum annum, Coriandrum sativum, Dolichos lablab, Lycopersicon esculentum, Solanum melongena, S. tuberosum, Vigna sinensis and Zingiber officinale and 5 fruits viz., Citrus aruntifolia, Coccus nucifera, Musa paradisiaca, Punica granatum and Pyrus malus were found in all months. Nineteen vegetables appeared only in winter, 21 only in summer and 30 in both winter and summer seasons but not throughout the year. Similarly, 11 fruits were available in winter, 9 in summer and 8 fruits occurred in both seasons but not throughout the year. Forty seven vegetables and 14 fruits were cultivated locally and 31 vegetables and 5 fruits were procured from other places. Fourteen vegetables and 8 fruits were brought to the market from wild habitats. Vegetables and fruits supplied from Hilly area and Terai plains were also noted. The most expensive vegetable and fruit were Mushrooms and pomegranate, respectively. Vegetable prices started to decrease from December and remained low during January, February and March; started to increase from April and reached at climax in May and June. Generally, the price of fruits was high from April to July.
\end{abstract}

Key words: Vegetable, fruit, Gundruk, Yangben, edible ferns, price survey, Dharan

\section{Introduction}

Vegetables and fruits are essential for human health as they provide energy, protein, vitamins and minerals. In Nepal, cereals provide larger amount of energy (i.e., 88\%) in daily diet. Due to health awareness and important nutritive values of vegetables and fruits, the per capita consumption of vegetables in Nepal has increased from $49 \mathrm{~kg} /$ person/year/ to 60 $\mathrm{kg} /$ person/year, but remains still below the human vegetable nutritional requirement i.e., $104 \mathrm{~kg} /$ person/year (HMG/N, 2006). Malnutrition is a major public health problem in Nepal and its main cause is our cereal based diet (Rai et al., 2002). Several kinds of epidemic diseases are caused due to nutrient deficiency. The most significant nutritional disorder is Protein-Energy Malnutrition, Vitamin A deficiency, Iodine Deficiency Disorders (IDD) and Fe deficiency anemia (Rai et al., 2002).

The history of development of vegetables in Nepal dates back in early forties; however, its rapid development was started from 1972 when Vegetable Development Division (VDD) 
was established in the Department of Agriculture (Awasthi, 2003). At present, there are more than 247 cultivated vegetable crops throughout the world and more than 50 vegetable crops are common in Nepal. Due to wide range of climatic conditions ranging from tropical to alpine temperate, it is possible to grow almost all types of vegetables in Nepal. The average productivity of vegetables and fruits in Nepal has varied between 9-12 MT/ha over the last decade. The productivity of vegetables has increased from 9.5 to 12.2 MT/ha between 1998 and 2007 whereas the productivity of fruits fluctuated between 10.5 and 9.5 MT/ha during the same year (Kakra \& Bhattacharjee, 2009).

Vegetables are less risky, fast growing and best sources of income in comparison to other cereal crops. Due to the market access and increasing demand of vegetables, area for cultivation and productivity of vegetables is also increasing over the years. According to the data of Kalimati Fruits and Vegetable Market Development Board, locally produced and internally supplied vegetables were $87 \%$, while only $13 \%$ was imported from India and others countries. Before 15 years the local production met only $13 \%$ demand and the rest was imported. An efficient market information system can play a vital role to increase the income of producer farmers and regularize supply in the market. Commercialization of vegetable cultivation requires establishing markets and trading centers with adequate storage facilities close to farmer's production centers.

Dharan (Lat. $26^{\circ} 49^{\prime} \mathrm{N}$ and Long. $87^{\circ} 16^{\prime} \mathrm{E}$; Elevation $349 \mathrm{msl}$ ) is a beautiful and multicultural city situated at the foothills of the Mahabharat range in Sunsari district, eastern Nepal. It is a trading centre for vegetables and fruits as it lies at the junction of hilly region and the Terai plains. It is the largest and most well-developed wholesale vegetable markets in the eastern Nepal. Generally, rainy season vegetables are procured here from north hilly districts (viz., Dhankuta, Bhojpur, Sunsari, Terathum and Sankhuwashawa) to supply to the south Terai plain of Nepal and India, as the production is very negligible in Terai due to heavy rainfall and waterlogged condition. Just opposite, in winter, the products are imported here from terai and India to supply into the hilly areas. The major vegetables traded in this market are tomato, chili, cabbage, cucumber and cauliflower (USAID, 2011). Many local people are engaged in this business for maintaining their livelihood.

Literature reveal that Balakrishnan et al. (1981), Sangwan (1989) and Jordan et al. (1998) have studied the prices of potatoes in Indian markets. Similarly, Thirupathi (1997) and Babu (2002) have studied the marketing and price of fruits in India. In context of Nepal, such study has not been carried out before, except some works in Kathmandu valley. An attempt has been made to survey the vegetable and fruit markets in Dharan to understand the seasonal and monthly availability and fluctuations in the rate of prices throughout the year.

\section{Methodology}

Extensive survey of vegetables and fruits sold in the local market of Dharan Municipality was carried out for one year from October, 2011 to September, 2012. Vegetable and fruit markets were visited on the first day of every month to know the availability, types and prices of vegetables and fruits present. For this, five permanent shopkeepers were selected for questionnaire. Information about commonly available vegetables and fruits, their rout of supply, current prices etc., were recorded. 


\section{Results}

In the present study, 77 vegetables belonging to 58 genera and 28 families and 33 fruits belonging to 20 genera and 16 families were recorded in one year from Dharan including their average monthly prices (Tab. 1).

The largest genus of vegetable was Brassica with 7 species followed by Capsicum 3 sp., Cucumis $3 \mathrm{sp}$. and so on and the largest family was Cucurbitaceae with 20 plants followed by Brassicaceae and Fabaceae 10 plants each, Solanaceae 8 plants and so on. Similarly, the largest genus of fruit was Citrus with 9 species followed by Pyrus $3 \mathrm{sp}$. and so on and largest family was Rutaceae with 10 plants followed by Rosaceae 5 plants and so on.

Eleven vegetables viz., Agaricus bisporus, Allium cepa, A. sativum, Capsicum annum, Coriandrum sativum, Dolichos lablab, Lycopersicon esculentum, Solanum melongena, S. tuberosum, Vigna sinensis and Zingiber officinale and 5 fruits viz., Citrus aruntifolia, Coccus nucifera, Musa paradisiaca, Punica granatum and Pyrus malus were present in all the months throughout the year in Dharan (Tabs. 1 and 2). Nineteen vegetables (Allium cepa leaf, A. sativum leaf, Alocasia indicum, Benicasa hispida, Brassica oleraceae, B. rapa, Chenopodium album, Colocassia antiquorum, Cuccurbita moschata, Cyclanthera pedata, Dioscorea alata, D. hamiltoni, Ipomoea batatus, Lathyrus sativus Lepidium sativum, Manihot esculenta, Pisum sativum, Sechium edule root and Vicia fava) appeared only in winter season. Similarly, 21 vegetables (Amanita vaginata, Artrocarpus heterophyllus, Astraeus hygrometricus, Bauhinia variegata, Capsicum frutescens var. grossum, Crateva unilocularis, Cucumis melo var. utilissimus, Cucurbita maxima, Dryopteris cochleata, D. splendens, Ficus lacor, Luffa acutangula, Moringa oleifera, Musa paradisiaca spathe, Ophioglossum vulgatum, young shoots of Sechium edule, Smilax aspera, Tamarindus indica, Tectaria macrodonta, Tinospora cordifolia and Trichosanthes anguina) were seen only in the summer. Thirty vegetables were found both in winter and summer season but not throughout the year were Abelmoschus esculentus, Agaricus campestris, Amaranthus caudatus, Anthum sowa, Bambusa tulda, Beta vulgaris, Brassica campestris B. juncea, B. junceae var folicosa, B. oleracea var. botrytis, B. oleraceae var capatata, Capsicum frutesens var. conoides, Colocasia esculenta, Corchorus capsularis, Cucumis sativus, Cucurbita maxima, Cyphomandra betaca, Daucas carota var. sativa, Fagopyrum esculentum, Glycine max green pod, Lagenaria siceraria, Luffa cylindrica, Momordica charantia, Raphanus sativa, Rorippa nasturtium-aquaticum, Sechium edule, Spinacia oleraceae var inerrus, Trichosanthes dioca, Trigonella foenum-graceum and Urtica dioca. Brassica was the winter vegetable but except B. rapa, all were available in the market in both seasons.

Vagetables available in the market for maximum period of 10 months were Agaricus campestris, Brassica juncea var. folicosa, B. oleraceae var capitata, Raphanus sativa and Rorippa nasturtium-aquaticum; those available for 9 months were Amaranthus caudatus, Brassica oleracea var. botrytis, Cucumis sativus and Daucas carota var. sativa; vegetables available for 8 months were Capsicum frutesens var. conoides, Cucurbita maxima, Momordica charantia, Sechium edule and Urtica dioca; and those available for 7 months were Anthum sowa, Cyphomandra betaca, Lagenaria siceraria and Trichosanthes dioca. Ophioglossum vulgatum was seen in the market for only one month whereas Amanita 
vaginata, Astraeus hygrometricus, Bauhinia variegata, Brassica rapa, Crateva unilocularis, Cucumis melo var. utilissimus and Ficus lacor were seen for two months only.

Similarly, 11 fruits (Citrus aurantium, C. junos, C. limettioides, C. maxima, $C$. medica, $C$. recticulata, $C$. sinensis, Rhus parviflora, $R$. semialata, Zizyphus jujube and Z. mauritiana) were found only in winter, 9 fruits (Ananas comosus, Citrullus lanatus, Litchi chinensis, Mangifera indica, Prunus domestica, P. domestica sub sp. instiata, Schleichera oleosa, Syzygium cumini and Trapa bispinosa) were found only in summer and 8 fruits (Annona squamosa, Carica papaya, Citrus lemon, Phyllanthus emblica, Psidium guajava, Pyrus communis, P. pashia and Vitis vinifera) were found in both seasons but not throughout the year. The winter is the season for Citrus as cultivated fruits and Rhus and Zizyphus as wild fruits.

The fruits available in the market for maximum period of 7 months was Phyllanthus emblica; followed by Citrus recticulata (6 months); Ananas comosus, Carica papaya, Citrus lemon, Psidium guajava, Vitis vinifera (5 months) and so on. Trapa bispinosa was available in the market for only one month. Similarly, Citrus junos, $C$. limettioides, C. maxima, $C$. medica, Prunus domestica sub. sp. instiata, Schleichera oleosa, Syzygium cumini and Zizyphus jujuba lasted in the market for two months.

There were about 47 vegetables (onion leaf, garlic leaf, amaranthus leaf, Indian dil, jack fruit, bamboo shoot, tuberous gourd, Indian rape, leaf mustard, colocasia leaf, co-co-yam, coriander, cucumber, tender parts of pumpkin shoot, vegetable marrow, young pumpkin, yam, hyacinth bean, buck-wheat, sweet potato, bottle gourd, peper cres, tomato topioca cassava, bitter gourd, drum-stick, banana flower, pea, radish, brinjal, potato, fenu-greek, broad bean, cow pea, ginger etc.) and 14 fruits (papaya, pommelo pompelmous, litchi, mango, banana, plum etc) produced and supplied from nearby areas in and around Dharan and 31 vegetables and 5 fruits were imported from outside. Fourteen vegetables (padkey chayau, devre chayau, tusa tama, koiralo, karkalo, bantarul, rani neuro, danthe neuro, kavro, jibre sag, sim sag, kukur daino, kali neuro, sisnoo) and 8 fruits (amala, mayel, bhakki amilo, kusum, jamun, satibyar, kasibayer, bayer) were brought to the market from wild habitat.

The vegetables generally supplied from Hills were bamboo shoot, camel's foot, mustard leaf, cauliflower, cabbage, turnip, capsicum chilly, cherry pepper, coriander leaf, garlic pear, cucumber, young pumpkin, ripen pumpkin, tree tomato, yam, garden pea, hyacinth bean, dauthe neuro, common fig, soyabean pod, sweeet potato, peper cres, tomato, chatel gourd, adder's tongue, radish, tender parts of chyote christopine, chyote christopine, green briers, brinjal, potato, kali neuro, gurjo, stinging nettle, stinging flower, broad bean, cow pea, ginger, caygua, edible fern shoot, padkey chayau, devra chayau and the fruits were lime, lemon otaheite, sweet lime, citron, loose skinned orange, Nepal sweet orange, litchi, plum, pear, Nepal sumac, Chinese sumac and mayel.

Similarly, the vegetables generally supplied from Terai were lady's finger, onion bulb, onion leaf, giant taro, beet, cauliflower, brocauli, cabbage, squash, capsicum chilly, bethe sag, white jute sag, coriander leaf, snake cucumber, carrot, hyacinth bean, bottle gourd, grass-pea, ridge ghiroula, tomato, bitter gourd, drum stick, radish, tamarind, snake gourd, pointed gourd, cow pea and cultivated mushrooms. The fruits imported from Terai were pineapple, custard apple, papaya, watermelon, coconut, litchi, mango, banana, Indian 
gooseberry, black plum, singhara nut, grapes and Indian plum. Apple was supplied from Jomsom (Western part of Nepal), and from India. Other fruits supplied from India were mango, grapes, seville orange, pomegranate, jujube fruit, Indian plum etc.

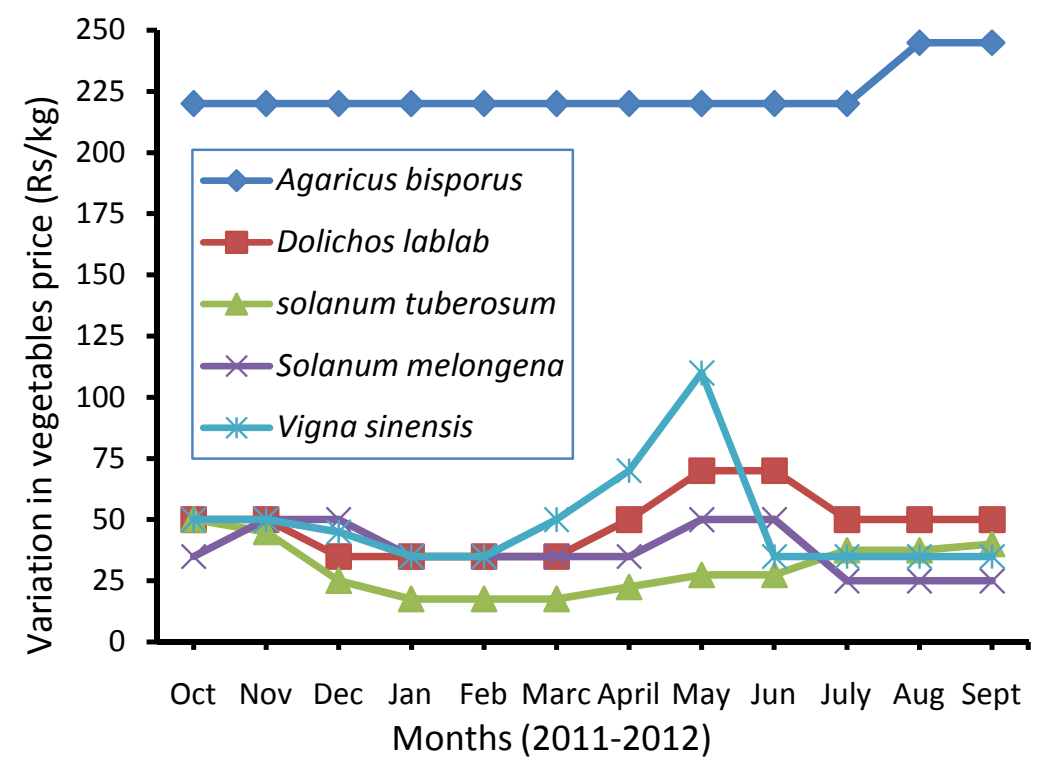

Figure 1. Monthly variations in the price of five major vegetables (found throughout the year) in Dharan.

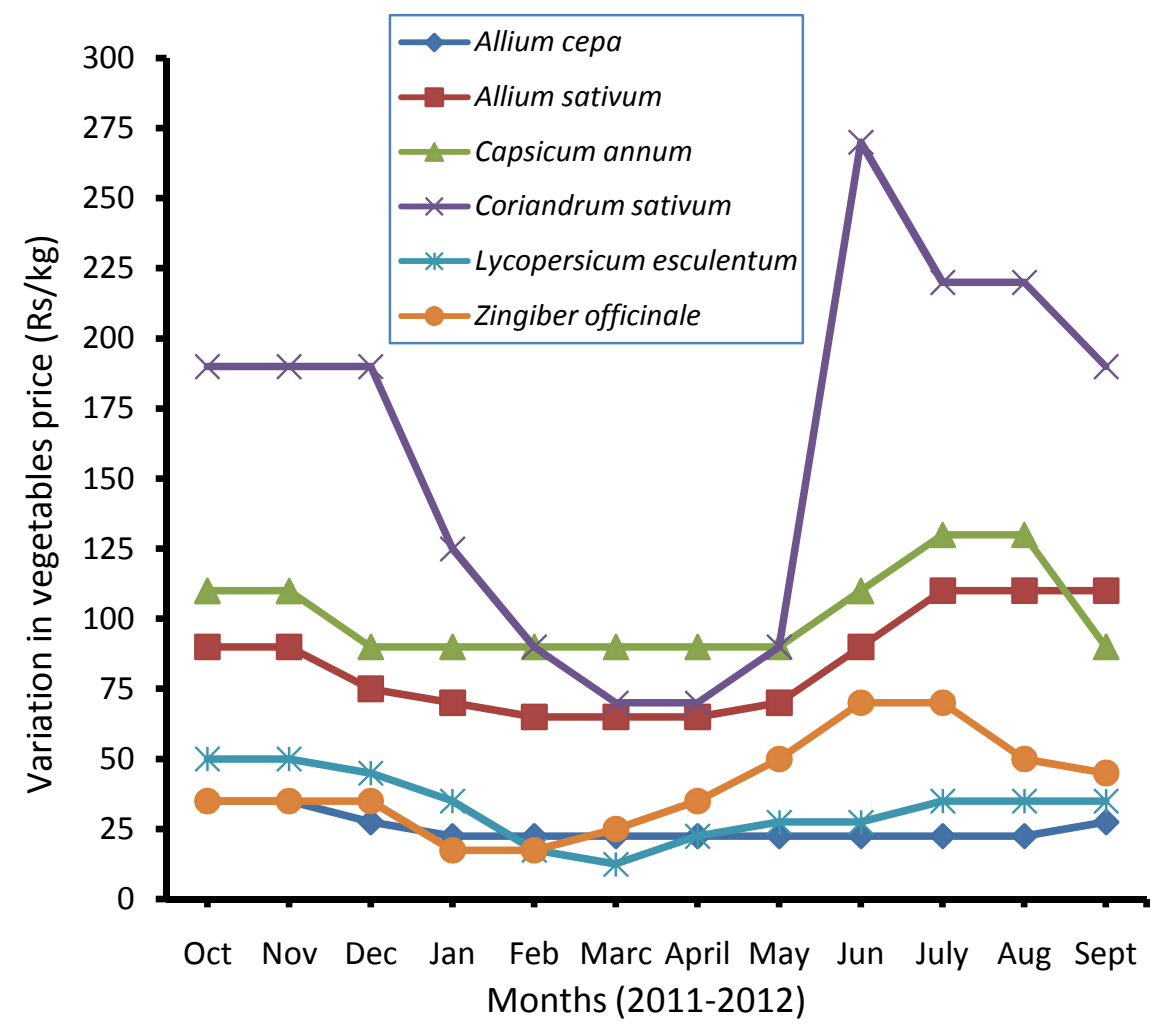

Figure 2. Monthly variations in the price of six minor vegetables (found throughout the year) in Dharan. 
The expensive vegetables were the Mushrooms viz. Amanita vaginata, Agaricus campestris, Astraeus hygrometricus and Agaricus bisporus whose prices/kg were Rs. 750, 500, 450 and 245 respectively (Tab. 1). Stinging nettle's flower and fruits were packed and sold at Rs. $500 / \mathrm{kg}$ and its green leafy shoot at Rs $280 / \mathrm{kg}$. The common trend in variation of prices of major vegetables was that the price started to decrease in December; remained low during January, February and March; started to raise in April and reached at climax in May and June (Fig. 1). For minor vegetables, the trend of price variation was quite different. Price started to decrease in September; remained low during January to April; then started to increase in May and reached climax in July and August (Fig. 2). Monthly variation on the price of coriander leaf was distinct but Agaricus bisporus and Allium cepa did not followed the general trend of variation.

Among the fruits, the expensive one was pomegranate which was 290/kg in the months of May, October and November (Tab. 2). It was followed by apple (240/kg in July), grape (180/kg in April), mango (100/kg in September), pineapple (80/kg in September) and so on. Generally, the price of fruits was high from April to July but in other months there was no regular pattern in variation of prices (Fig. 3).

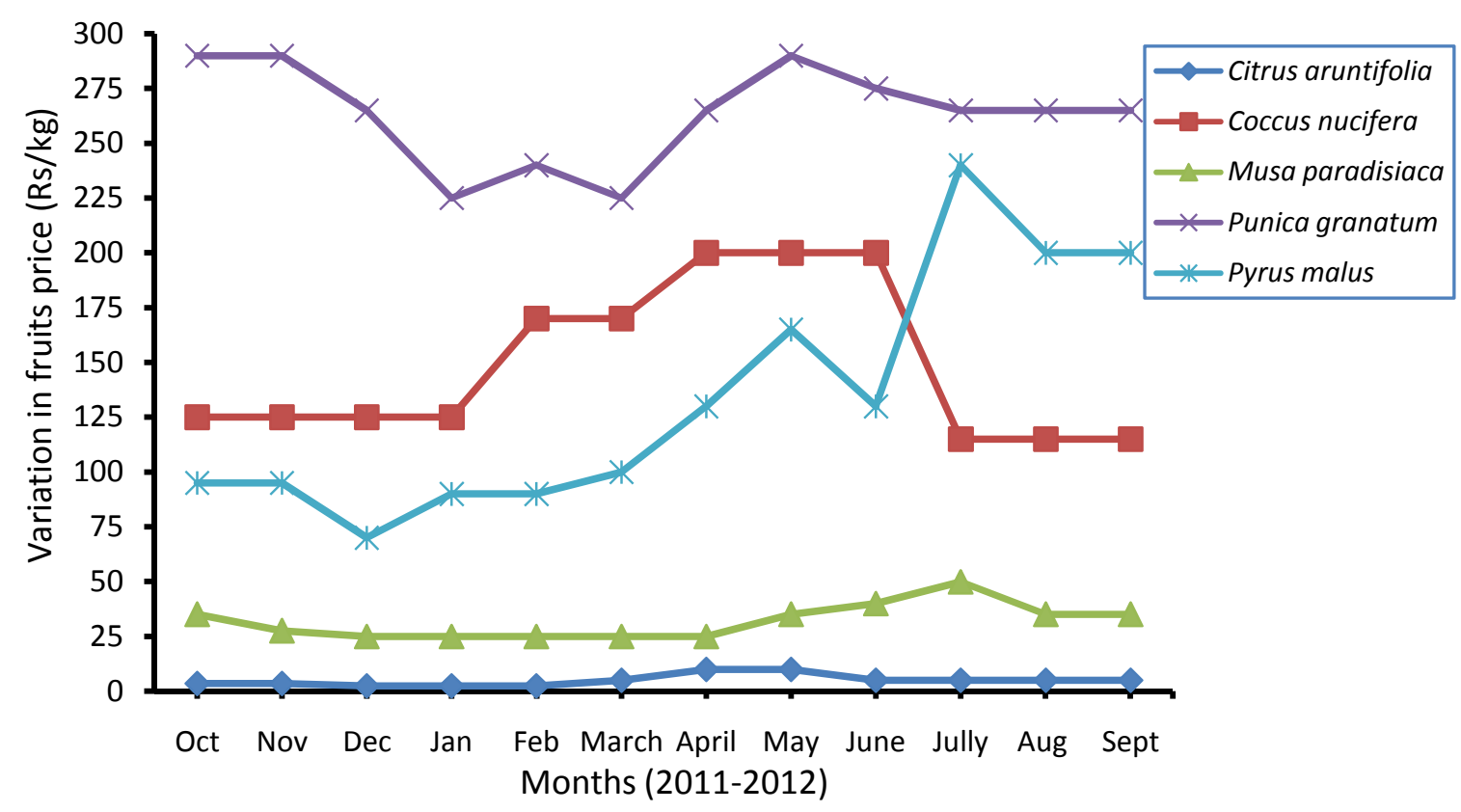

Figure 3. Monthly variations in the price of five fruits (found throughout the year) in Dharan.

\section{Discussion}

Advances in agronomy and developments in transport system have made every variety of vegetables and fruits available all year-round, although unseasoned vegetables and fruits do not taste as good as the seasonal ones (Shrestha, 1983).

Dharan is largest centre for fresh and delicious vegetables and fruits varieties. As vegetables and fruits are imported here both from terai and hills, most of these are available throughout 
Table 1. Vegetables sold in the market of Dharan with their monthly price variations (2011/2012).

\begin{tabular}{|c|c|c|c|c|c|c|c|c|c|c|c|c|c|c|c|}
\hline \multirow{2}{*}{ SN } & \multirow{2}{*}{ Vegetables } & \multirow{2}{*}{ Common name } & \multirow{2}{*}{ Local name } & \multirow{2}{*}{ Family } & \multicolumn{11}{|c|}{ Price $(\mathrm{Rs} / \mathrm{kg})$} \\
\hline & & & & & Oct & Nov & Dec. & Jan $\mathbf{I}$ & Feb 1 & MarApr I & May & Jun. & July & Aug & g Sep \\
\hline 1 & $\begin{array}{l}\text { Abelmoschus esculentus (L.) } \\
\text { Moench. }\end{array}$ & Lady's finger & Bhindi & Malvaceae & 70 & - & - & - & - & 90 & 70 & 32.5 & 25 & 25 & 50 \\
\hline 2 & Agaricus bisporus (Lange) Pitat & Cultivated mushroom & Chate chayau & Agaricaceae & 220 & 220 & 2202 & 2202 & 220 & 220220 & 220 & 220 & 220 & 245 & 245 \\
\hline 3 & A. campestris $\mathrm{L}$. & Cultivated mushroom & Dallay chayau & Agaricaceae & 500 & 500 & 500 & 4504 & 450 & 450450 & 450 & 450 & 450 & - & - \\
\hline \multirow[t]{2}{*}{4} & Allium cepa $\mathrm{L}$. & Onion bulb & Pyaj & Amaryllidaceae & 35 & 35 & 27.52 & 22.52 & 22.5 & 22.522 .52 & 22.5 & 522.5 & 22.5 & 22.5 & 527.5 \\
\hline & & Onion leaf & Pyaj ko pat & Amaryllidaceae & - & - & 25 & 25 & 35 & $35-$ & - & - & - & - & - \\
\hline \multirow[t]{2}{*}{5} & A. sativum $\mathrm{L}$. & Garlic bulb & Lashun & Amaryllidaceae & 90 & 90 & 75 & 70 & 65 & $65 \quad 65$ & 70 & 90 & 110 & 110 & 110 \\
\hline & & Garlic leaf & Lashun ko pat & Amaryllidaceae & - & - & 50 & 50 & 35 & $45-$ & - & - & - & - & - \\
\hline 6 & Alocasia indicum (Roxb.) Schott. & Giant Taro & Oal & Araceae & - & - & - & 50 & 50 & $35-$ & - & - & - & - & - \\
\hline 7 & Amanita vaginata (Bull.) Lam. & Grisette & Devre chayau & Amanitaceae & - & - & - & - & - & - & - & 750 & 750 & - & - \\
\hline 8 & Amaranthus caudatus L. & Amaranthus leaves & Latte sag & Amaranthaceae & 35 & 35 & 50 & 50 & 50 & - & - & 25 & 25 & 25 & 35 \\
\hline 9 & Anthum sowa Roxb. & Indian dil & Sounp sag & Umbelliferae & 65 & 65 & 454 & 42.53 & 32.5 & $35 \quad 35$ & - & - & - & - & - \\
\hline 10 & Artrocarpus heterophyllus Lam. & Jack fruit & Rukh katahar & Moraceae & - & - & - & - & - & $-\quad 45$ & 35 & 27.5 & 35 & 32.5 & $5-$ \\
\hline 11 & $\begin{array}{l}\text { Astraeus hygrometricus (Pers.) } \\
\text { Morgan }\end{array}$ & Hygroscopic Earthstar & Padkey chayau & Agaricaceae & - & - & - & - & - & - & 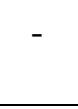 & 450 & 450 & - & - \\
\hline 12 & Bambusa tulda Roxb. & Bamboo shoot & Tusa tama & Poaceae & 50 & 70 & - & - & - & - & - & 70 & 50 & 32.5 & 535 \\
\hline 13 & Bauhinia variegata $\mathrm{L}$. & Camels foot & Koiralo & Fabaceae & - & - & - & - & - & $-\quad 95$ & 70 & - & - & - & - \\
\hline 14 & Benicasa hispida (Thunb.) Cogn. & Tuberous gourd & Kuvindo & Cucurbitaceae & - & 45 & 45 & 45 & - & - & - & - & - & - & - \\
\hline 15 & Beta vulgaris $\mathrm{L}$. & Beet & Guliyo moola & Chenopodiaceae & - & - & 95 & 95 & 85 & $85 \quad 85$ & - & - & - & - & - \\
\hline 16 & Brassica campestris $\mathrm{L}$. & Indian rape & Tori sag & Brassicaceae & - & - & 45 & 35 & 35 & $35 \quad 20$ & - & - & - & - & - \\
\hline 17 & B. juncea (Rupr.) Sinskaya & Leaf mustard & Rayo duku sag & Brassicaceae & - & - & 65 & 55 & 55 & 47.537 .5 & - & - & - & - & - \\
\hline 18 & B. junceae var folicosa Bail. & Broad leaf mustard & Rayo sag & Brassicaceae & 55 & 55 & 47.53 & 37.53 & 37.5 & $25 \quad 35$ & 35 & - & - & 50 & 55 \\
\hline
\end{tabular}




\begin{tabular}{|c|c|c|c|c|c|c|c|c|c|c|c|c|c|c|c|c|}
\hline 19 & B. oleracea var. botrytis L. & Cauliflower & Phulkopi & Brassicaceae & 75 & 67.5 & 35 & 22.5 & 22.5 & 20 & - & - & - & 90 & 90 & 75 \\
\hline 20 & B. oleraceae var capitata L. & Cabbage & Banda kopi & Brassicaceae & 35 & 35 & 22.5 & 22.5 & 11.51 & 11.5 & - & -2 & 22.5 & 17.5 & 17.5 & 17.5 \\
\hline 21 & B. oleraceae var. italica L. & Broccoli & Brocauli & Brassicaceae & - & 75 & 50 & 53 & 27.52 & 22.5 & - & - & - & - & - & - \\
\hline 22 & B. rapa $\mathrm{L}$. & Turnip & Salgam & Brassicaceae & - & 80 & 70 & - & - & - & - & - & - & - & - & - \\
\hline 23 & Capsicum annum $\mathrm{L}$. & Capsicum, Chilly & Khorsani & Solanaceae & 110 & 110 & 90 & 90 & 90 & 90 & 90 & 90 & 110 & 130 & 130 & 90 \\
\hline 24 & C. frutesens L. var. conoides Bailey & Cherry pepper & Dallay , & Solanaceae & 440 & 450 & 290 & 290 & 340 & 340 & - & - & - & - & 440 & 440 \\
\hline 25 & C. frutescens L. var. grossum Bailey & Bull-nose chilly & $\begin{array}{l}\text { Bhende } \\
\text { khursani }\end{array}$ & Solanaceae & - & - & - & - & - & - & 90 & 100 & 100 & 100 & 110 & - \\
\hline 26 & Chenopodium album L. & Bethe & Bethu & Chenopodiaceae & - & - & 85 & 70 & 70 & 70 & - & - & - & - & - & - \\
\hline 27 & $\begin{array}{l}\text { Colocasia antiquorum Schott. var. } \\
\text { esculenta L. }\end{array}$ & $\begin{array}{l}\text { Co-Co, Yam, Taro, } \\
\text { Arum }\end{array}$ & Pindalu & Araceae & - & - & 25 & 25 & 27.5 & 35 & - & - & - & - & - & - \\
\hline & C. esculenta (L.) Schott & Colocassia leaf & Karkalo & Araceae & 50 & 50 & - & - & - & - & - & - & - & - & - & 50 \\
\hline & Corchorus capsularis L. & White jute & Patuwa sag & Tiliaceae & - & - & - & - & - & 35 & 35 & 35 & 35 & - & - & - \\
\hline 30 & Coriandrum sativum L. & Coriander & Dhaniya leaf & Umbelliferae & 190 & 190 & 190 & 125 & 90 & 70 & 70 & 90 & 270 & 220 & 220 & $\overline{190}$ \\
\hline & Crateva unilocularis Buch.-Ham. & Garlic pear & Sipligan & Capparaceae & - & - & - & - & - & - & 90 & 90 & - & - & - & - \\
\hline & Cucumis sativus L. & Cucumber & Pahade kakro & Cucurbitaceae & 50 & - & - & - & - & - & - & - & 75 & 50 & 27.5 & 32.5 \\
\hline & & Cucumber & Kakro & Cucurbitaceae & 35 & 50 & 50 & - & - & - & 70 & 65 & 27.5 & 27.5 & 22.5 & 22.5 \\
\hline 33 & $\begin{array}{l}\text { C. melo L. var. utilissimus Duth. \& } \\
\text { Full. }\end{array}$ & Snake cucumber & Kakri & Cucurbitaceae & - & - & - & - & - & - & - & 32.5 & 35 & - & - & - \\
\hline & Cucurbita maxima D. Don & $\underline{\text { Leafy shoot }}$ & Pharsi munta & Cucurbitaceae & - & - & - & - & - & - & 95 & 95 & 45 & 45 & 45 & - \\
\hline & & Vegetable marrow & Pharsi ko phul & Cucurbitaceae & - & - & - & - & - & - & - & 45 & 45 & 45 & - & - \\
\hline & & Young pumpkin & Gatta & Cucurbitaceae & 25 & 25 & 25 & 35 & 35 & 35 & 50 & - & - & - & - & 27.5 \\
\hline & & Ripen pumpkin & Pharsi & Cucurbitaceae & - & - & - & - & - & - & - & 35 & 25 & 22.5 & 27.5 & 27.5 \\
\hline 35 & $\begin{array}{l}\text { C. moschata (Duchesne ex Lam.) } \\
\text { Duchesne ex Poiret }\end{array}$ & Squash & Guliyo pharsi & Cucurbitaceae & - & - & 35 & 35 & 35 & 25 & - & - & - & - & - & - \\
\hline
\end{tabular}




\begin{tabular}{|c|c|c|c|c|c|c|c|c|c|c|c|c|c|c|c|}
\hline 36 Cyclanthera pedata (L.) Schrader & Caygue & Chuchey kareli & aCucurbitaceae & 70 & 50 & 35 & 35 & 35 & 35 & - & - & - & - & - & - \\
\hline 37 Cyphomandra betaca (Cav.) Sendt. & Tree tomato & Rukh bhenta & Solanaceae & 70 & 70 & 60 & 45 & 35 & 35 & - & - & - & - & - & 70 \\
\hline 38 Daucas carota L. var. sativa DC. & Carrot & Gazar & Umbelliferae & 65 & 55 & 27.5 & 27.5 & 22.5 & 17.5 & - & - & - & 140 & 110 & 90 \\
\hline 39 Dioscorea alata L. & White yam & Ghartarul & Dioscoreaceae & - & - & - & 55 & 45 & 55 & - & - & - & - & - & - \\
\hline 40 D. hamiltoni Hook. F. & Yam & Bantarul & Dioscoreaceae & - & - & - & 95 & 85 & 75 & - & - & - & - & - & - \\
\hline 41 Dolichos lablab L. & Hyacinth bean & Tate simi & Fabaceae & 50 & 50 & 35 & 35 & 35 & 35 & 50 & 70 & 70 & 50 & 50 & 50 \\
\hline 42 Dryopteris cochleata (D. Don) & Edible fern shoot & Rani neuro & Aspidiaceae & - & - & - & - & - & - & - & 57.5 & 45 & 25 & 20 & 20 \\
\hline 43 D. splendens (Hook.) Kuntze & Wild fern & Danthe neuro & Aspidiaceae & - & - & - & - & - & - & - & - & - & 57.5 & 57.5 & 50 \\
\hline 44 Fagopyrum esculentum Moench & Buck- wheat & Phapar sag & Polygonaceae & - & - & 75 & 75 & 75 & 50 & 50 & - & - & - & - & - \\
\hline 45 Ficus lacor Buch-Ham. & Common fig & Kavro & Moraceae & - & - & - & - & - & - & 95 & 80 & - & - & - & - \\
\hline 46 Glycine $\max (\mathrm{L}$.$) Merr.$ & Soyabean pod & Bhatmas kosa & Fabaceae & 50 & - & - & - & - & - & - & - & - & - & 35 & 35 \\
\hline 47 Ipomoea batatus (L). Lam. & Sweet potato & Suthuni & Convolvulaceae & - & - & - & 45 & 45 & 45 & - & - & - & - & - & - \\
\hline 48 Lagenaria siceraria (Molina) Standl. & Bottle gourd & Lauka & Cucurbitaceae & 27.5 & - & - & - & - & -1 & 22.5 & 22.5 & 17.5 & 17.5 & 17.5 & 17.5 \\
\hline 49 Lathyrus sativus L. & Grass-pea & Khesari sag & Fabaceae & - & - & - & 37.5 & 35 & 35 & - & - & - & - & - & - \\
\hline 50 Lepidium sativum L. & Peper cres & Chamsur sag & Brassicaceae & - & 72.5 & 72.5 & 50 & 35 & 35 & - & - & - & - & - & - \\
\hline 51 Luffa acutangula (L.) Roxb. & Ridge gourd & Pate ghiroula & Cucurbitaceae & - & - & - & - & - & - & - & 90 & 50 & 35 & 35 & 50 \\
\hline 52 L. cylindrica (L.) Roem. & Sponge gourd & Ghiroula & Cucurbitaceae & 50 & - & - & - & - & - & 90 & 70 & 45 & 25 & 30 & 35 \\
\hline 53 Lycopersicum esculentum Mill. & Tamato & Tamatar & Solanaceae & 50 & 50 & 45 & 35 & 17.5 & $12.5^{\prime}$ & 22.5 & 27.52 & 27.5 & 35 & 35 & 35 \\
\hline 54 Manihot esculenta Crantz. & Topioca, Cassava & Simal tarul & Euphorbiaceae & - & - & - & 30 & 27.5 & 30 & - & - & - & - & - & - \\
\hline 55 Momordica charantia L. & Bitter gourd & Tite karela & Cucurbitaceae & 50 & 50 & - & - & - & - & 90 & 70 & 25 & 25 & 25 & 35 \\
\hline 56 M. cochinchinensis Spr. & Chathel gourd & Chatel & Cucurbitaceae & 90 & - & - & - & - & - & - & - & - & - & 90 & 90 \\
\hline 57 Moringa oleifera Lam. & Drumstick & Sahijan & Moringaceae & - & - & - & - & - & - & 90 & 70 & 60 & - & - & - \\
\hline 58 Musa paradisiaca L. & Banana flower & Bungo & Musaceae & - & - & - & - & - & - & - & - & - & 35 & 35 & 35 \\
\hline 59 Ophioglossum vulgatum L. & Adders tongue & Jibre sag & Ophioglossaceae & - & - & - & - & - & - & - & - & - & 65 & - & - \\
\hline
\end{tabular}




\begin{tabular}{|c|c|c|c|c|c|c|c|c|c|c|c|c|c|c|c|c|}
\hline \multirow[t]{2}{*}{60} & \multirow[t]{2}{*}{ Pisum sativum L. } & Garden pea & Kerau sag & Fabaceae & - & - & 35 & 35 & 35 & 35 & - & - & - & - & - & - \\
\hline & & Garden pea & Kerau kosa & Fabaceae & - & - & - & 27.5 & 25 & 25 & - & - & - & - & - & - \\
\hline 61 & Raphanus sativa L. & Radish & Moola & Brassicaceae & 40 & 30 & 22.5 & 22.5 & 17.5 & 11.5 & 11.5 & - & - & 35 & 22.5 & 17.5 \\
\hline 62 & $\begin{array}{l}\text { Rorippa nasturtium-aquaticum (L) } \\
\text { Hayek. }\end{array}$ & Water cress & Sim sag & Brassicaceae & 35 & 35 & - & - & 50 & 50 & 50 & 50 & 22.52 & 22.5 & 27.5 & 35 \\
\hline \multirow[t]{3}{*}{63} & \multirow[t]{3}{*}{ Sechium edule (Jacq.) Sw. } & Leafy shoot & Ishkus munta & Cucurbitaceae & - & - & - & - & - & - & 95 & 95 & 45 & 45 & 45 & 50 \\
\hline & & Chyote Christophine & Ishkus & Cucurbitaceae & 17.5 & 17.5 & 12.5 & 12.5 & 17.5 & 22.5 & - & - & - & - & 22.5 & 17.5 \\
\hline & & Chyote Christophine & Ishkus ko jara & Cucurbitaceae & 85 & 80 & 70 & 55 & 55 & 55 & - & - & - & - & - & - \\
\hline 64 & Smilax aspera L. & Green briers & Kukur daino & Liliaceae & - & - & - & - & - & - & - & - & 85 & 85 & - & - \\
\hline 65 & Solanum melongena L. & Brinjal & Baigun & Solanaceae & 35 & 50 & 50 & 35 & 35 & 35 & 35 & 50 & 50 & 25 & 25 & 25 \\
\hline 66 & S. tuberosum L. & Potato & Aloo & Solanaceae & 50 & 45 & 25 & 17.5 & 17.5 & 17.5 & 22.52 & 27.5 & 27.53 & 37.5 & 37.5 & 40 \\
\hline 67 & Spinacia oleraceae var inerrus L. & Round seeded spinach & Palungo sag & Chenopodiaceae & - & - & 85 & 85 & 42.5 & 42.5 & 42.5 & 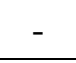 & - & - & - & - \\
\hline 68 & Tamarindus indica $\mathrm{L}$. & Tamarind & Titri & Fabaceae & - & - & - & - & - & - & - & - & 90 & 90 & - & - \\
\hline 69 & Tectaria macrodonta (Fee.) C.Chr. & Wild fern & Kali neuro & Aspidiaceae & - & - & - & - & - & - & -6 & 62.5 & 45 & 35 & 32.5 & - \\
\hline 70 & Tinospora cordifolia (Wild.)Miers. & Gulancha tinospora & Gurjo & Menispermaceae & - & - & - & - & - & - & - & - & 65 & 65 & - & - \\
\hline 71 & Trichosanthes anguina L. & Snake gourd & Chichindo & Cucurbitaceae & - & - & - & - & - & - & - & 50 & 25 & 25 & 25 & 35 \\
\hline 72 & T. dioca Roxb. & Pointed gourd & Parwar & Cucurbitaceae & 50 & - & - & - & - & - & 90 & 70 & 32.5 & 30 & 35 & 50 \\
\hline 73 & Trigonella foenumgraceum L. & Fenunagreek & Methi sag & Fabaceae & - & - & 55 & 55 & 55 & 55 & 55 & - & - & - & - & - \\
\hline \multirow[t]{2}{*}{74} & \multirow[t]{2}{*}{ Urtica dioca $\mathrm{L}}$. & Stinging nettle & Sisnoo & Urticaceae & 140 & 220 & 280 & 280 & 280 & 280 & - & - & - & - & 110 & 140 \\
\hline & & Stinging nettle flower & Sisnoo phool & Urticaceae & 180 & 260 & 500 & 500 & - & - & - & - & - & - & - & 180 \\
\hline 75 & Vicia fava $\mathrm{L}$. & Broad bean & Bakula simi & Fabaceae & - & - & 70 & 70 & 50 & 50 & - & - & - & - & - & - \\
\hline 76 & Vigna sinensis (L.) Savi ex Hassk. & Cow pea & Tane bodi & Fabaceae & 50 & 50 & 45 & 35 & 35 & 50 & 70 & 110 & 35 & 35 & 35 & 35 \\
\hline & Zingiber officinale Rosc. & Ginger & Aduwa & Zingiberaceae & 35 & 35 & 35 & 17.5 & 17.5 & 25 & 35 & 50 & 70 & 70 & 50 & 45 \\
\hline
\end{tabular}

- = Not available 
Table 2. Edible fruits sold in the market of Dharan with their monthly price variations (2011/2012).

\begin{tabular}{|c|c|c|c|c|c|c|c|c|c|c|c|c|c|c|}
\hline \multirow{2}{*}{ SN } & \multirow{2}{*}{ Common name } & \multirow{2}{*}{ Local name } & \multirow{2}{*}{ Family } & \multicolumn{11}{|c|}{ Price (NRs/Kg) } \\
\hline & & & & Oct & Nov & Dec & Jan & Feb & Mar & Apr 1 & - May & y June & July & Aug Sep \\
\hline 1 Ananas comosus (L.) Merr. & Pineapple & Bhui katar & Bromeliaceae & - & - & - & - & - & - & - & 55 & 50 & 55 & 6580 \\
\hline 2 Annona squamosa $\mathrm{L}$. & Custard apple & Sarifa & Annonaceae & 75 & - & - & - & - & - & - & - & - & - & $75 \quad 75$ \\
\hline 3 Carica papaya $\mathrm{L}$. & Papaya & Mewa & Caricaceae & - & - & 35 & 35 & 27.5 & 27.52 & 27.5 & - & - & - & - \\
\hline $\begin{array}{l}4 \text { Citrullus lanatus (Thunb.). } \\
\text { Mat. \& Nakai }\end{array}$ & Watermelon & Tarbuja & Cucurbitaceae & - & - & - & - & - & - & 35 & 35 & 37.5 & - & - \\
\hline $\begin{array}{l}5 \text { Citrus aruntifolia (Christ.) } \\
\text { Swinge }\end{array}$ & Lime & Kagati & Rutaceae & $3.5 / \mathrm{p}$ & $3.5 / p$ & $2.5 / \mathrm{p}$ & $2.5 / \mathrm{p}$ & $2.5 / \mathrm{p}$ & $5 / \mathrm{p}$ & $10 / p$ & $10 / p$ & $5 / p$ & $5 / \mathrm{p}$ & $5 / \mathrm{p} \quad 5 / \mathrm{p}$ \\
\hline 6 C. aurantium L. & Seville orange & Mausam & Rutaceae & 85 & 85 & 85 & - & - & - & - & - & - & - & - \\
\hline 7 C. junos Sieb.ex Tanaka & Yuzu lemon & Jyaamir & Rutaceae & - & $12.5 / \mathrm{p}$ & $15 / \mathrm{p}$ & - & - & - & - & - & - & - & - \\
\hline 8 C. lemon (L.) Burn.f. & Lemon (yellow) & Nibua & Rutaceae & $10 / \mathrm{p}$ & $10 / \mathrm{p}$ & $10 / \mathrm{p}$ & $10 / \mathrm{p}$ & - & - & - & - & - & - & $-10 / \mathrm{p}$ \\
\hline 9 C. limettioides Tanaka. & Sweet lime & Chaksi & Rutaceae & - & $22.5 / \mathrm{p}$ & $22.5 / \mathrm{p}$ & - & - & - & - & - & - & - & - \\
\hline 10 C. maxima (Burm.) Herr. & Pommelo pompelmou & s Bhogate & Rutaceae & - & $17.5 / \mathrm{p}$ & $12.5 / \mathrm{p}$ & - & - & - & - & - & - & - & - \\
\hline 11 C. medica $\mathrm{L}$. & Citron & Bimiro & Rutaceae & - & $22.5 / p$ & $22.5 / \mathrm{p}$ & - & - & - & - & - & - & - & - \\
\hline 12 C. recticulata Blanco. & loose skinned orange & Suntala & Rutaceae & 65 & 65 & 50 & 50 & 75 & 95 & - & - & - & - & - \\
\hline 13 C. sinensis Osbeck. & Nepal sweet orange & Junar & Rutaceae & - & 95 & 95 & 95 & - & - & - & - & - & - & - \\
\hline 14 Coccus nucifera $\mathrm{L}$. & Coccunut & Nariwal & Arecaceae & 125 & 125 & 125 & 125 & 170 & 170 & 200 & 200 & 200 & 115 & 115115 \\
\hline 15 Litchi chinensis Sonner. & Litchi & Licchi & Sapindaceae & - & - & - & - & - & - & -1 & $17.5 / \mathrm{d}$ & $\mathrm{d} 17.5 / \mathrm{d} 2$ & 122.5/d & $d-$ \\
\hline 16 Mangifera indica $\mathrm{L}$. & Mango & Amp & Anacardiaceae & - & - & - & - & - & - & - & - & 50 & 60 & $70 \quad 100$ \\
\hline 17 Musa paradisiaca L. & Banana & Kera & Musaceae & $35 / \mathrm{d}$ & $27.5 / \mathrm{d}$ & $25 / \mathrm{d}$ & $25 / \mathrm{d}$ & $25 / d$ & $25 / \mathrm{d}$ & $25 / d$ & $35 / \mathrm{d}$ & $40 / \mathrm{d}$ & $50 / \mathrm{d}$ & $35 / \mathrm{d} \mathrm{35/c}$ \\
\hline
\end{tabular}




\begin{tabular}{|c|c|c|c|c|c|c|c|c|c|c|c|c|c|c|c|}
\hline 18 Phyllanthus emblica L. & Indian gooseberry & Amala & Euphorbiaceae & 35 & 35 & 27.5 & 22.5 & 22.5 & 22.5 & - & - & - & - & - & 35 \\
\hline 19 Prunus domestica L. & Plum & Alu bakhara & Rosaceae & - & - & - & - & - & - & - & 62.5 & 55 & 70 & - & - \\
\hline $\begin{array}{l}20 \text { P. domestica L. sub. sp. } \\
\quad \text { instiata (L.) }\end{array}$ & Bullace & Alucha & Rosaceae & - & - & - & - & - & - & - & 45 & 45 & - & - & - \\
\hline 21 Psidium guajava L. & Guava & Amba & Myrtaceae & 60 & 45 & 35 & 35 & - & - & - & - & - & - & - & 60 \\
\hline 22 Punica granatum L. & Pomegranate & Anar & Punicaceae & 290 & 290 & 265 & 225 & 240 & 225 & 265 & 290 & 275 & 265 & 265 & 265 \\
\hline 23 Pyrus communis L. & Pear & Naspati & Rosaceae & 35 & - & - & - & - & - & - & - & - & 27.5 & 35 & 35 \\
\hline 24 P. malus L. & Apple & Syau & Rosaceae & 95 & 95 & 70 & 90 & 90 & 100 & 130 & 165 & 230 & 240 & 200 & 200 \\
\hline 25 P. pashia Ham.ex D.Don & Himalayan pear & Mayal, Mel & Rosaceae & 35 & 35 & - & - & - & - & - & - & - & - & - & 35 \\
\hline 26 Rhus parviflora Roxb. & Nepal sumac & Satibyar & Anarcardiaceae & - & - & 45 & 50 & 50 & 50 & - & - & - & - & - & - \\
\hline 27 R. semialata Murr. & Chinese sumac & Bhakki amilo & Rutaceae & - & $12.5 / \mathrm{p}$ & $12.5 / \mathrm{p}$ & $12.5 / \mathrm{p}$ & - & - & - & - & - & - & - & - \\
\hline $\begin{array}{l}28 \text { Schleichera oleosa (Lour.) } \\
\text { Oken }\end{array}$ & $\begin{array}{l}\text { Lac tree, Macassar oil } \\
\text { tree, Ceylon oak }\end{array}$ & Kusum & Sapindaceae & - & - & - & - & - & - & - & - & - & 60 & 70 & - \\
\hline 29 Syzygium cumini (L.) Skeels & Black Plum & Jamun & Myrtaceae & - & - & - & - & - & - & - & - & - & 75 & 90 & - \\
\hline 30 Trapa bispinosa Roxb. & Singhara nut & Pani singhara & Trapaceae & - & - & - & - & - & - & - & 35 & - & - & - & - \\
\hline 31 Vitis vinifera L. & Grapes & Angur & Vitaceae & - & - & 95 & 105 & 130 & 140 & 180 & - & - & - & - & - \\
\hline 32 Zizyphus jujuba P. Mill. & Jujube fruit & $\begin{array}{l}\text { Kasibayer } \\
\text { Narkeli ruit }\end{array}$ & Rhamnaceae & - & - & - & - & 85 & 90 & - & - & - & - & - & - \\
\hline 33 Z. mauritiana Lam. & Indian plum, & Bayer & Rhamnaceae & - & - & 35 & 35 & 25 & 22.5 & 5 & - & - & - & - & - \\
\hline
\end{tabular}

$\mathrm{p}=$ piece, $\mathrm{d}=$ dozen, - = Not available 
the year. In hills cauliflower and cabbage and also brinjals are largely grown in the early winter season and these are largely supplied in mid winter to the end of the season. It is seen that delicious hilly potatoes and tomatoes are largely supplied in summer seasons and as winter arises, terai tomatoes and potatoes are seen with the prices in descending order till the end of winter and also till the early summer season. Due to flooding in terai the rate is increased but as monsoons ends up the prices goes on descending order.

Winter is the season for fresh Citrus fruits and fresh vegetables. These Citrus fruits are very essential as they contain vitamin $\mathrm{C}$ that helps our body to fight against germs. Many vegetables and fruits are winter crops but also they are found whole year around. This is because of cold store facilities and also supply and availability from both terai and hilly regions throughout the year.

Summer vegetable and fruits are soft skinned and moisture filled and perfectly suited for hot climates. Most of the red and yellow vegetables and fruits contain beta-carotene, an antioxidant. It is known to increase immunity and prevent cancer disease. Greens are very good source of vitamin A, B6, C, riboflavin and folate and rich in minerals such as calcium, iron, magnesium, phosphorus, potassium, zinc and manganese.

Varieties of chilies like bird's eye chilli, jire khursani (Capsicum microcarpum DC.); bell pepper, bhende khursani (Capsicum frutescens var. grossum Bailey); long pepper, lapche khursani (Capsicum frutescens var. verbasciculatum Bailey); cone pepper, ghokre khursani (Capsicum frutescens var. conoides Bailey); cone pepper, chuchhe khursani (Capsicum frutescens var. conoides Bailey) were found in the market. The fermented vegetable items like 'sinki' prepared from radish, 'gundruk' made from mustard and radish leaf, sour taste tusa tama from bamboo bud, 'kinema' from soyabean and 'yengben' (lichen) were also found occasionally in Dharan.

Due to availability of junk foods, the demand of fresh vegetables and fruits are declining day by day in today's busy lives. On the contrary, vegetables and fruits are the only two food constituents that when taken on a regular basis can keep all kinds of diseases away. Various organic and inorganic fertilizers are used to increase the quantity and quality of fruits and vegetables so it is advisable to rinse all vegetables and fruits thoroughly before intake.

For wholesale market, September and October is the best season for wholesale prices, and February and March is the worst. Cucumber, onion and chili are in better price positions than tomato, cabbage and cauliflower (USAID, 2011). Farmers of this region who are able to produce off-season vegetables from May to November would get premium prices. There is fluctuation of rates due to various causes like, if there is heavy inpute from the sources, the rate decreases and if there are transportation strikes, the rate increases.

\section{Acknowledgements}

Authors are thankful to the Head, Department of Biology, Central Campus of Technology, Dharan for laboratory space. Thanks are due to Mr. Kuber Shrestha for continuous encouragement and help to complete this work. 


\section{References}

Awasthi, B.D. 2003. Vegetable production and marketing in Kathmandu valley. Community Rural Development Society, Devinagar, Kathmandu.

Babu, J.D., R.S. Babu, V. Shankaraiah \& B.G. Singh. 2002. An analysis of grading and its influence on marketing of custard apple fruits. J. Research ANGRAU 30(2): 30-32.

Balakrishnan, V., L.P. Swaminathan \& V. Puhazhendhi. 1981. An analysis of prices and arrivals of potato in Nilgiri district of Tamil Nadu. Agricultural Marketing 23(4): 1-4.

Chaudary, R.P. 1998. Biodiversity in Nepal. Tec Press, Bangkok.

Dangol, D.R. 2005. Dictionary of forest and common land plants of western Chitwan. Institute for Social and Environmental Research, Nepal, Fulbari, Chitwan, Nepal.

Hara, H., A.O. Charter \& L.H. Williams. 1982. An enumeration of the flowering plants of Nepal. Vol. III. British Museum (Nat. Hist.), London.

Hara, H., W.T. Stern \& L.H. Williams. 1978. An enumeration of the flowering plants of Nepal. Vol. I. British Museum (Nat. Hist.), London.

HMG/N. 2006. Statistical information on Nepalese agriculture. Agri-Busines Promotion and Statistics Division, MOAC. Singha Durbar, Kathmandu, Nepal. 23p.

Jordan, J.L., S.E. Prussia \& R.L. Shewfelt. 1998. A hedonic approach to estimating the value of quality characteristics of horticultural crops. Acta-Horticulturae 223(1): 376-382.

Kakra, A. \& N. Bhattacharjee. 2009. The impact of the global economic and financial crisis in Least Developed Countries' manufacturing industry: The case of the fruits and vegetables sector in Bhutan \& Nepal.100p.

Rai, S.K., K. Hirai, A. Abe \& Y. Ohno. 2002. Infectious Diseases and Malnutrition Status in Nepal: an Overview. Mal. J. Nutr. 8(2): 191-200.

Sangwan, S.S. 1989. Seasonal variation in potato price in important markets of the country. Ind. J. Agri. Mark. 3(1): 120-125.

Shrestha, K. 1983. Wild leafy and fruity vegetable consumed by the local inhabitants of Dharan. J. Nat. Hist. Mus. 7(1): 35-42.

Shrestha, K. 1998. Dictonary of Nepelese plant names. Natural History Museum, T.U., Kathmandu, Nepal.

Thirupathi, R.S. 1997. Role of grading and packing in price spread of apple fruit: A case study of Himachal Pradesh hills. Ind. J. Agri. Mark. 10(3): 20-25.

USAID. 2011. Nepal economic agriculture and trade activity-value chain/market analysis of the off-season vegetable sub-sector in Nepal. United States Agency for International Development, General Development Office, Kathmandu, Nepal. 45p. 